\title{
RESULTADOS DE ENSAYOS DEL BANCO AUTOMÁTICO DE ASPERSORES
}

\author{
Salvatierra Bellido, B. (1), Andrades Gonzalez, J.A(2), Gómez Durán, E. (3), \\ Viqueira Pina, S. (4).
}

\author{
${ }^{1}$ Tecnólogo titular, IFAPA Centro Chipiona, (Cádiz), benito.salvatierra@juntadeandalucia.es \\ ${ }^{2}$ Técnico Colaborador, IFAPA Centro Chipiona, (Cádiz). \\ ${ }^{3}$ Técnico Colaborador, IFAPA Centro Chipiona, (Cádiz). \\ ${ }^{4}$ Técnico I+D+F, IFAPA Centro Chipiona, (Cádiz), salvador.viqueira@juntadeandalucia.es
}

\section{Resumen.}

Desde el IFAPA Centro Chipiona como integrante del Sistema de Asesoramiento al Regante de la Junta de Andalucía, se da servicio a las zonas del bajo Guadalquivir y costeras de la provincia de Cádiz donde más profusamente se emplean los sistemas de riego por aspersión. Las características del riego por aspersión y la variabilidad de su eficiencia según las condiciones climáticas y de diseño, así como el alto número de variables ajustables en el manejo, alimentaron la necesidad de crear un sistema con el que ensayar el comportamiento de los diferentes aspersores frente a múltiples escenarios de viento. El objeto último del ensayo es dar respuesta esta necesidad para ello se creó en el IFAPA Centro Chipiona el primer banco automático de ensayo anemométrico de aspersores, presentado en el XXXII Congreso Nacional de Riegos, mediante el cual se ensayan las diferentes combinaciones de aspersión dentro de una matriz pluviométrica, tomándose registro de las condiciones climáticas para crear posteriormente escenarios de modelado, donde obtener la combinación de mayor eficiencia para las condiciones de diseño que se planteen.

\section{1- Introducción y objetivos}

Como primer sistema de riego se ha querido ensayar un aspersor de impacto circular, con doble boquilla, por ser uno de los más empleados en cobertura total por los usuarios del Sistema de Asesoramiento al Regante de nuestra zona del Bajo Guadalquivir y de la zona Regable de la Costa Noroeste de Cádiz.

Este trabajo tiene como objetivo presentar al sector los primeros resultados sobre el comportamiento del aspersor ensayado en el banco automático de aspersores del IFAPA centro Chipiona.

Como primer sistema de riego se ha querido ensayar un aspersor de impacto circular, con opción de doble boquilla, por ser uno de los más empleados en aspersión en la zona del Bajo Guadalquivir y de la zona Regable de la Costa Noroeste de Cádiz.

Se procedió al ensayo de los diferentes conjuntos de aspersión, hasta conseguir una muestra de una hora de viento estable estudiados para cada uno de los rangos.

\section{2- Materiales y método.}

Se eligió el aspersor de impacto con barrido circular de doble boquilla f46 de UNIRAIN.

Se eligen los complementos del aspersor:

- Boquillas: Se han probado diferentes boquillas para el aspersor ensayado. Los diferentes diámetros se identifican fácilmente por un código de colores.

- Vainas: Se ensayan las boquillas con vainas prolongadoras del chorro o sin ésta. 
Se procedió a realizar en ensayo del aspersor para diferentes situaciones de presión, alta, media y baja, $\left(2,5 \mathrm{~kg} / \mathrm{cm}^{2}, 3,5 \mathrm{~kg} / \mathrm{cm}^{2}, 4,5 \mathrm{~kg} / \mathrm{cm}^{2}\right)$ la presión se fija en el cabezal mediante la actuación de una válvula de esfera.

Se agrupan los datos de viento en episodios donde el Vector _Viento se mantenga constante, considerándose como muestra episodios de una hora en los que no se varía dirección ni velocidad más de un $10 \%$. Los datos del vector viento, se recogen mediante una anemoveleta conectada al sistema. Se realiza una medición en continuo, registrando el dato medio de la variable cada 10 segundos, siendo nuestra muestra final las mediciones de una hora de viento estable, tendrá por tanto 360 mediciones registradas por cada situación.

Los sistemas de riego de aspersión suelen trabajar a una velocidad de viento en el rango de 0-4 metros por segundo. Se puede considerar que vientos superiores a cuatro metros por segundo conllevan una distorsión en la distribución del agua por parte del aspersor tan acentuada que sería imposible evaluar la tendencia de esta sobre el aumento en la velocidad del viento. Para analizar a la pluviometría del sistema ensayado es necesario escenarios muestrales estables, por tanto consideraremos como nulos aquellos ensayos en los que exista viento racheado. De este modo clasificaremos los vientos en 4 tipos dependiendo de su velocidad.

- Leve: cuando la velocidad media del viento no supera los $2 \mathrm{~m} / \mathrm{s}$

- Moderado: cuando la velocidad media del viento supera los $2 \mathrm{~m} / \mathrm{s}$ pero no sobrepasa la velocidad de $4 \mathrm{~m} / \mathrm{s}$

- Fuerte: la velocidad del viento sobrepasa los $4 \mathrm{~m} / \mathrm{s}$

- Racheado: la desviación típica de la media de velocidad de viento durante el ensayo es mayor de $20^{\circ}$ y su velocidad media supera los $4 \mathrm{~m} / \mathrm{s}$.

Dentro y en cada uno de los tipos de velocidades de viento, se incluirá la variable "Dirección del viento" (DV) que es la dirección de ataque del viento respecto a las líneas de aspersión que conforman los marcos posteriormente simulados con la ayuda del programa "SpacePro" en el cual podremos modificar el ángulo de entrada del viento. Tomando como referencia la Figura 1, se pueden considerar tres tipos de direcciones:

- Viento paralelo a la línea de aspersión: vector de velocidad media del viento forma ángulo cero respecto a la línea de cultivo, con una desviación típica no mayor de $20^{\circ}$.

- Viento oblicuo a la línea de aspersión: vector de velocidad media del viento durante el ensayo forma un ángulo de 45 grados respecto a la línea de aspersión, con una variabilidad de $20^{\circ}$.

- Viento perpendicular a la línea de aspersión: vector de velocidad media del viento perpendicular a la línea de aspersión, con una variabilidad de $20^{\circ}$.

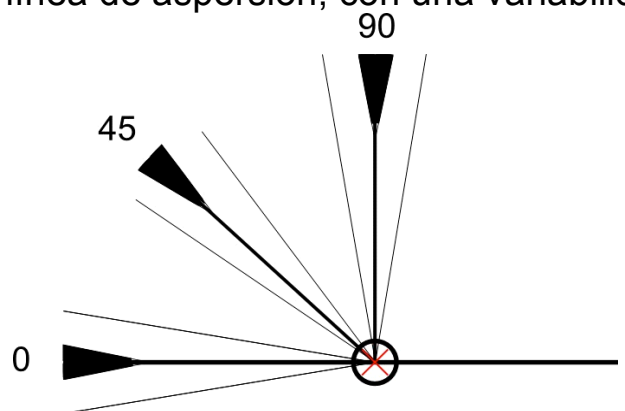

Figura 1: Representación gráfica de las direcciones descritas

La captación de los datos de pluviometría se realiza mediante una matriz pluviométrica. Nos dará la distribución espacial de los $\mathrm{mm} / \mathrm{h}$. Los datos medidos se acumulan en un datalogger para su posterior procesado. Se procede a la recogida de los datos a través de un programa informático compatible.

Recogidos los datos se procede a su análisis. Obtenemos una distribución pluviométrica media para cada episodio Viento, del aspersor aislado. 
La figura $n^{\circ} 2$ Muestra distribución de la pluviometría a partir de datos de la matriz de pluviométrica, para el aspersor ensayado con unas condiciones de viento concretas.

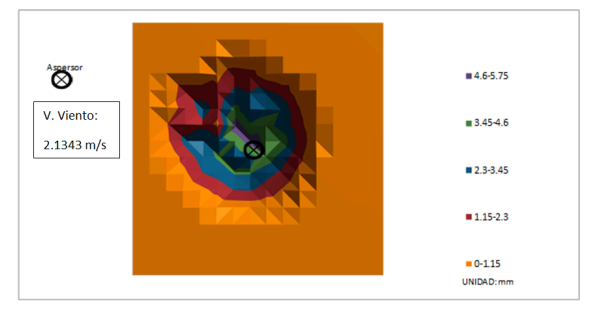

Figura $n^{\circ} 2$ : Distribución de la pluviometría.

Establecido el comportamiento del aspersor aislado, introducimos la variable marco. Procesaremos los datos obtenidos en cada uno de los ensayos a través del "SpacePro" para obtener los resultados de cada tipo de marco y dimensiones de este. Para la obtención de los resultados de este proyecto introduciremos en el "SpacePro" la variante de marco rectangular y marco triangular con dimensiones de 10x10 hasta $18 \times 18$ para ambos tipos de marco. De esta forma logramos saber cuáles son las uniformidades de distribución (DU) para cada boquilla y presión que se ha ensayado.

Extrapolamos matemáticamente mediante el programa informático "SpacePro" el comportamiento de cada marco para viento perpendicular, paralelo y oblicuo. Obtenemos la uniformidad de distribución de lluvia para la situación ensayada.

Para las evaluaciones de los ensayos de riego se utilizó la metodología propuesta por el Sistema de Asistencia al Regante de Andalucía del IFAPA (Salvatierra, 2012). El índice utilizado para caracterizar la uniformidad de las instalaciones en esta metodología es la Uniformidad de Distribución, UD. Se puede obtener el coeficiente de uniformidad de Christiansen a través de la fórmula:

\section{CUC $=((1-0.63 *(1-U D / \mathbf{1 0 0})) * 100 \quad$ (Merrian y Keller, 1978).}

La uniformidad de distribución (UD) se define como la relación entre la medida de mínima altura interceptada y la altura media interceptada.

Hay que tener en cuenta que optando por una uniformidad alta, se estará eligiendo una instalación de riego que ahorrará agua e incrementará la producción de los cultivos, lo que siempre es necesario. Pero también será mayor el coste de la instalación ya que las presiones deberán ser más uniformes, mayores los diámetros de tuberías, se necesitará mayor longitud de tubería, serán necesarios reguladores de presión, etc.

Así pues, habrá que llegar a una equilibrio, eligiendo una uniformidad lo suficientemente elevada que permita aprovechar el agua al tiempo que se obtienen buenas producciones, sin que el coste sea excesivo. En cualquier caso, la uniformidad de distribución deberá ser mayor o igual que el $75 \%$ (tabla 2 ).

Para abordar el análisis de los datos vamos a generar graficas que muestren el alcance de la uniformidad de distribución respecto a las distintas disposiciones de marco, fijando en cada caso las variables que correspondan.

Con objeto de extraer más fácilmente las conclusiones de los datos, se mantienen aquellas situaciones de marco y dirección de viento que reflejan el mismo caso. Es decir, la situación de marco 10x12 con dirección viento oblicuo respecto a las líneas de aspersión es la misma que marco $12 \times 10$ con viento oblicuo respecto a las líneas de aspersión. Igualmente la situación $12 \times 10$ con viento paralelo respecto a las líneas de aspersión es la misma que con marco $10 \times 12$ con viento perpendicular respecto a las líneas de aspersión

\section{3- Resultados.}

Debido a la gran cantidad de datos obtenidos y las múltiples combinaciones posibles que se pueden realizar, nos centraremos en procesar y analizar unos estudios en concreto. Para todos estos estudios hemos mantenido la presión como una constante, tomando como 
referencia una presión de 3,5 bar, exceptuando para el estudio 3 que se tomó una presión de 3,0 bar. Esta elección se ha tomado como consecuencia de que la gran mayoría de los riegos por aspersión en el bajo Guadalquivir varían entre los 3,0 bar y 3,5 bar.

Los estudios que hemos realizado son los siguientes:

- Estudio 1, Influencia de la dirección del viento: Variación de la UD respecto a las distintas disposiciones de marco para un ataque del viento paralelo, oblicuo y perpendicular. Variables fijadas: presión 3.5 bar y marco rectangular.

- Estudio 2, Influencia de la disposición del marco en cobertura superficial: Variación de la UD respecto a las distintas disposiciones de marcos pequeños según sea el marco rectangular o triangular. Variables fijadas: presión 3.5 bar y viento perpendicular.

- Estudio 3, Influencia de la disposición del marco en cobertura enterrada: Variación de la UD respecto a las distintas disposiciones de marco grandes según sea el marco rectangular o triangular. Variables fijadas: presión 3.0 bar y viento perpendicular.

- Estudio 4, Influencia de la velocidad del viento: Comparativa de la UD para viento fuerte y leve. Variables fijadas: viento oblicuo, marco rectangular y boquilla 5/32 VP.

Para abordar el análisis de los datos vamos a generar graficas que muestren el alcance de la uniformidad de distribución respecto a las distintas disposiciones de marco, fijando en cada caso las variables que correspondan.

Con objeto de extraer más fácilmente las conclusiones de los datos, se mantienen aquellas situaciones de marco y dirección de viento que reflejan el mismo caso. Es decir, la situación de marco 10×12 con dirección viento oblicuo respecto a las líneas de aspersión es la misma que marco $12 \times 10$ con viento oblicuo respecto a las líneas de aspersión. Igualmente la situación $12 \times 10$ con viento paralelo respecto a las líneas de aspersión es la misma que con marco $10 \times 12$ con viento perpendicular respecto a las líneas de aspersión.

\subsection{Estudio 1, Influencia de la dirección del viento}

El objetivo de este estudio es comparar y analizar como varía la uniformidad de distribución respecto a las distintas disposiciones de marco cuando el ataque del viento es paralelo, oblicuo o perpendicular. Usaremos para el estudio tres combinaciones diferentes de boquillas: la boquilla $1 / 8$ con vaina prolongadora, la boquilla $1 / 8$ con vaina prolongadora más boquilla secundaria $3 / 32$ y la boquilla $5 / 32$ con vaina prolongadora. Las variables que fijaremos serán la presión de trabajo que será de 3,5 bar y el tipo de marco que será rectangular.

\section{$>$ Boquilla 1/8" VP}

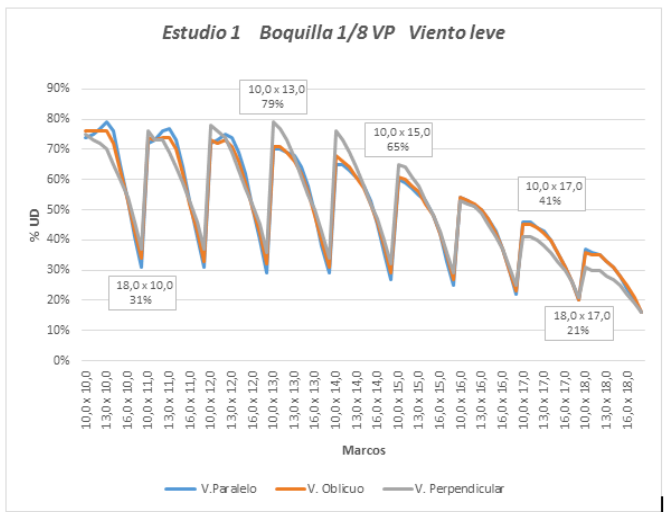


Gráfica 1: Gráfica lineal del porcentaje de uniformidad de distribución (UD) en diferentes tamaños de marco con tres tipos de dirección de ataque del viento: paralelo (a), oblicuo (b) y perpendicular (c) a las líneas de aspersión.

La uniformidad de distribución (UD) disminuye al aumentar el marco, y simultáneamente, cuanto más desproporcionado sea el rectángulo del marco (los dos lados de dimensiones muy desiguales (ejemplo: 18x10)) más drásticamente decrece la UD.

Se obtiene un mejor comportamiento con vientos perpendiculares en situaciones de marcos rectangulares moderados (dimensiones parecidas entre los dos lados) hasta valores de 15 en el lado de máxima longitud (ejemplo: 10x13).

En las direcciones de viento oblicuo y perpendicular se observa un comportamiento similar entre ellos, obteniendo valores inferiores a la dirección de viento perpendicular, exceptuando en marcos rectangulares desproporcionados con valores superiores al 16 en el lado de máxima longitud (ejemplo: 10x17).

Boquilla 1/8" VP + 3/32"

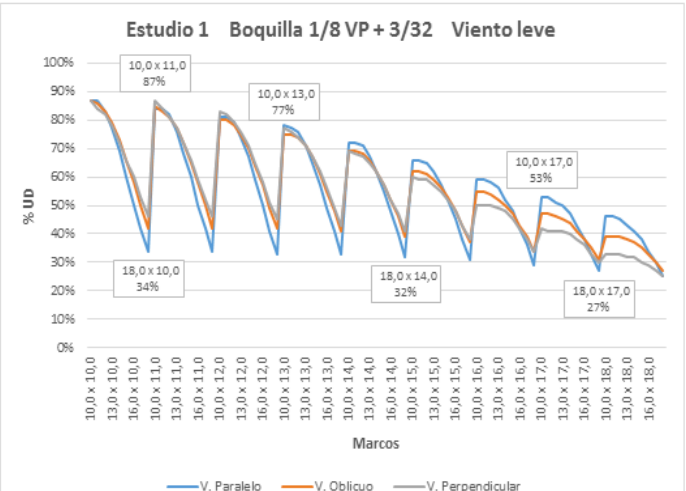

Gráfica 2: Gráfica lineal del porcentaje de uniformidad de distribución (UD) en diferentes tamaños de marco con tres tipos de dirección de ataque del viento: paralelo (a), oblicuo (b) y perpendicular (c) a las líneas de aspersión.

Se observa, al igual que ocurría en el caso anterior, como la uniformidad de distribución (UD) decrece al aumentar las dimensiones del marco, así como cuanto más desproporcionado sea el rectángulo del marco.

Se aprecia cómo los 3 tipos de dirección de ataque del viento tienen un comportamiento parecido, obteniéndose sus valores máximos de UD en marcos rectangulares moderados, donde el valor de uno de sus lados es 10 y el otro lado no sobrepasa el valor de 13 (ejemplo: 10x11).Sin embargo se distingue como a partir de valores superiores al 13 en el lado mayor del rectángulo, el valor de UD para viento perpendicular es inferior respecto al viento oblicuo, y éste a su vez es inferior respecto al viento paralelo (ejemplo: 10x17). 
Boquilla 5/32 VP

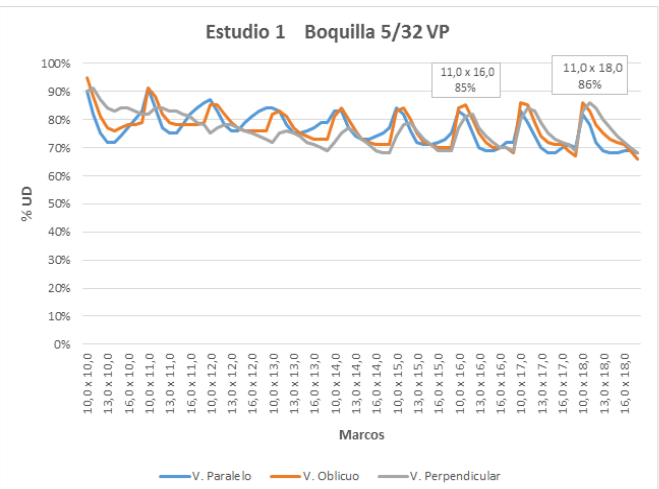

Gráfica 3: Gráfica lineal del porcentaje de uniformidad de distribución (UD) en diferentes tamaños de marco con tres tipos de dirección de ataque del viento: paralelo (a), oblicuo (b) y perpendicular (c) a las líneas de aspersión.

A diferencia con los dos casos anteriores, se aprecia como la disminución de la UD es inferior al aumentar el tamaño del marco, manteniéndose los valores de UD entre $65 \%$ y $90 \%$ en los 3 vientos.

\subsection{Estudio 2, Influencia de la disposición del marco en cobertura superficial.}

La finalidad de este estudio es comparar y analizar como varía la uniformidad de distribución según sea rectangular o triangular el marco, para marcos de menos de $15 \times 15$. Usaremos para el estudio tres combinaciones diferentes de boquillas: la boquilla $1 / 8$ con vaina prolongadora, la boquilla $1 / 8$ con vaina prolongadora más boquilla secundaria $3 / 32$ y la boquilla $5 / 32$ con vaina prolongadora. Las variables que fijaremos serán la presión de trabajo que será de 3,5 bar y la dirección de ataque del viento que será perpendicular.

Boquilla 1/8 VP

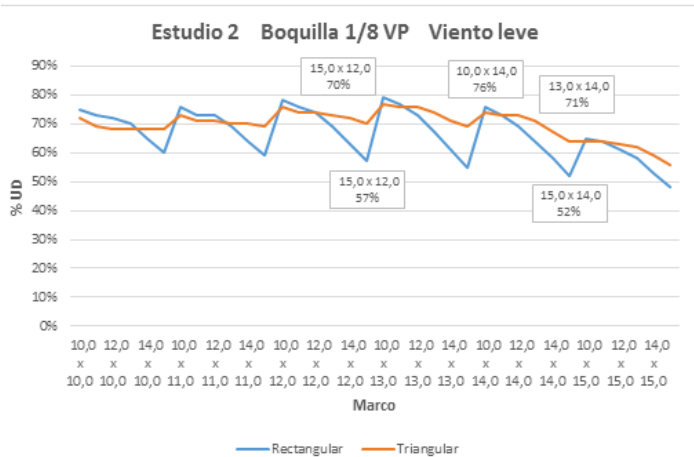

Gráfica 4: Gráfica lineal del porcentaje de uniformidad de distribución (UD) en diferentes tamaños de marco con dos tipos de dimensiones de marco: rectangular y triangular.

Se aprecia claramente como los valores de UD se mantienen más constantes, valores entre el $70-80 \%$, cuando el marco es triangular. No obstante, se observa como los valores de la uniformidad de distribución para marcos triangulares decrecen rápidamente, hasta valores inferiores al $60 \%$, conforme las dimensiones del marco aumentan a partir de marcos de $13 \times 14$.

Se observa la disminución de los valores de UD, para marcos rectangulares, cuando aumentan el tamaño de los marcos y conforme crecen las dimensiones de sus lados. 


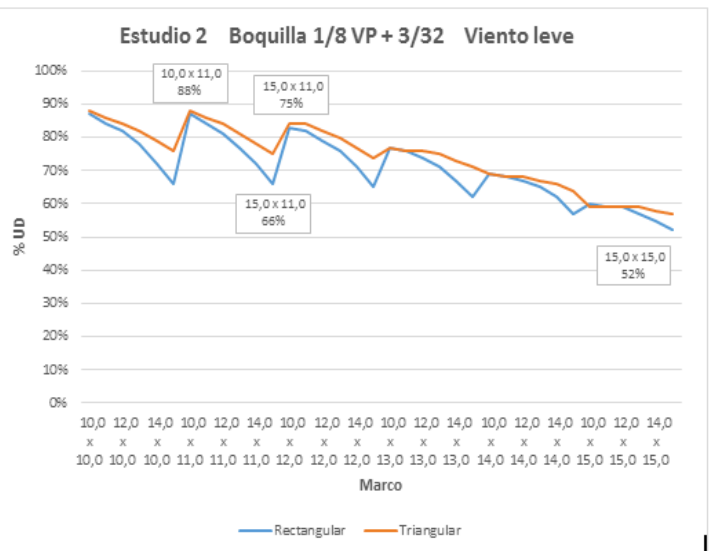

Gráfica 5: Gráfica lineal del porcentaje de uniformidad de distribución (UD) en diferentes tamaños de marco con dos tipos de dimensiones de marco: rectangular y triangular.

Se observa la disminución de los valores de UD según aumentan el tamaño de los marcos y conforme crecen las dimensiones de sus lados. Los valores de uniformidad de distribución disminuyen de forma similar tanto para marcos rectangulares como triangulares. Sin embargo, en marcos rectangulares este decrecimiento es más acentuado conforme aumenta el tamaño de uno de sus lados (ejemplo 15x11).

Boquilla 5/32 VP

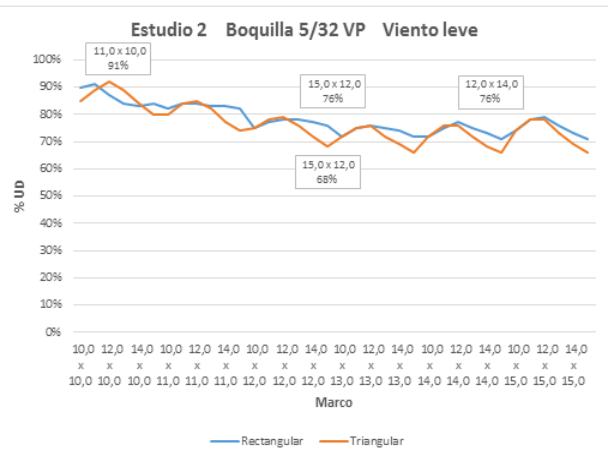

Gráfica 6: Gráfica lineal del porcentaje de uniformidad de distribución (UD) en diferentes tamaños de marco con dos tipos de dimensiones de marco: rectangular y triangular.

Se aprecia como los valores de uniformidad de distribución van disminuyendo según aumentan el tamaño de los marcos y conforme crecen las dimensiones de sus lados, aunque esta disminución es menos severa que la ocurrida en el caso de la boquilla 1/8 $\mathrm{VP}+3 / 32$.

Los valores de UD disminuyen de forma similar tanto para marcos rectangulares como triangulares. Sin embargo, en marcos triangulares este decrecimiento es más acentuado conforme aumenta el tamaño de uno de sus lados (ejemplo 15x12) a diferencia de lo que ocurría en el caso de la boquilla $1 / 8 \mathrm{VP}+3 / 32$, donde era el marco rectangulares el que acusaba más este decrecimiento de los valores de UD.

3.3. Estudio 3, Influencia de la disposición del marco en cobertura enterrada.

La finalidad de este estudio es comparar y analizar como varia la uniformidad de distribución según sea rectangular o triangular el marco, para marcos mayores de $15 \times 15$. Usaremos para el estudio dos combinaciones diferentes de boquillas: la boquilla 11/64 con vaina prolongadora y la boquilla 3/16 con vaina prolongadora. Las variables que fijaremos serán la 
presión de trabajo que será de 3,0 bar y la dirección de ataque del viento que será perpendicular.

\section{Boquilla 11/64}

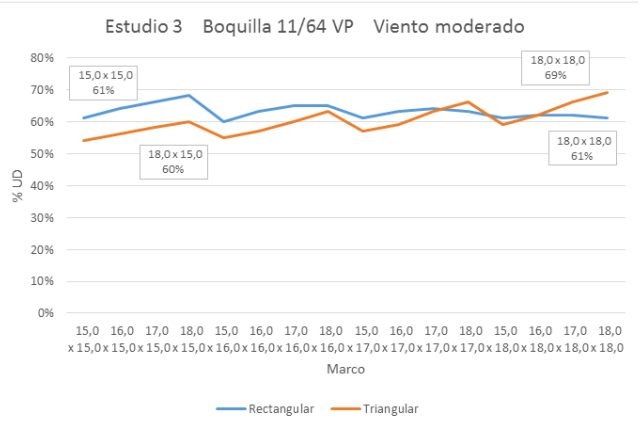

Gráfica 7: Gráfica lineal del porcentaje de uniformidad de distribución (UD) en diferentes tamaños de marco con dos tipos de dimensiones de marco: rectangular y triangular.

Se aprecia, en líneas generales, un mejor comportamiento de los valores de uniformidad de distribución en marcos rectangulares, exceptuando cuando ambos lados del marco son iguales o superiores a un valor de 17 (ejemplo 18x18)

Para marcos rectangulares los valores de UD se mantiene constantes conforme aumenta las dimensiones del marco, como se puede observar en los marcos $15 \times 15$ y 18x18 cuyos valores de UD son los mismos.

En marcos triangulares los valores de UD crecen según aumentan las dimensiones del marco, y simultáneamente al crecer el tamaño de uno de sus lados (ejemplo 18x15), alcanzando su máximo valor de UD en marco $18 \times 18$.

$>$ Boquilla 3/16 VP

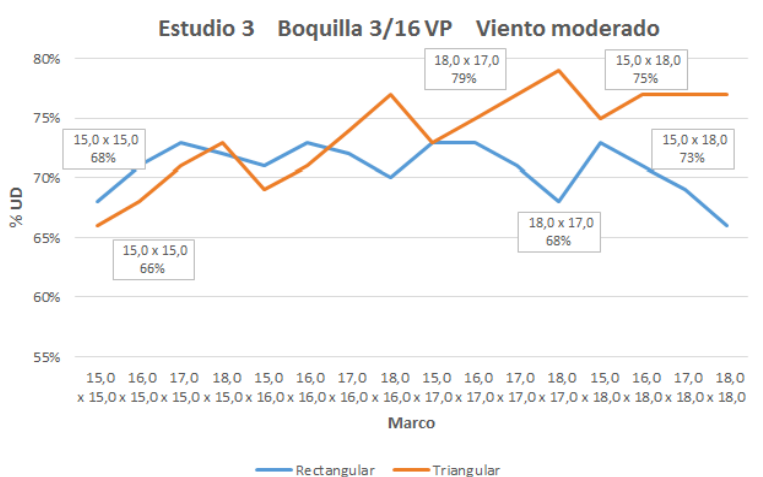

Gráfica 8: Gráfica lineal del porcentaje de uniformidad de distribución (UD) en diferentes tamaños de marco con dos tipos de dimensiones de marco: rectangular y triangular.

Se observa como para marcos triangulares los valores de uniformidad de distribución crecen al aumentar las dimensiones del marco y simultáneamente al crecer el tamaño de uno de sus lados. En marcos rectangulares se aprecia como los valores de UD se mantienen semejantes siempre que al menos uno de sus lados se mantenga en valores de 15. Estos valores disminuyen según aumenta el tamaño de uno de sus lados.

Como consecuencia de lo explicado en el apartado anterior, se puede observar en la gráfica como se obtienen mejores valores de UD para marcos triangulares exceptuando cuando las dimensiones del marco son mínimas (ejemplo 15x15) y valores similares, tanto en marco triangular como rectangular, cuando la dimensión de uno de sus lados en mínima (ejemplo 15x18). 


\subsection{Estudio 4, Influencia de la velocidad del viento.}

En este estudio pretendemos ver las fortalezas de una boquilla en dos situaciones de velocidad de viento. Para ello se fijan aquellas variables que aseguran un mejor funcionamiento para diferentes velocidades de viento, dichas condiciones están justificadas en ensayos de campos ya realizados por el SAR (Salvatierra, 2012). Según dichas condiciones para un viento fuerte se recomienda una presión de trabajo de 3,0 bar con objeto de aumentar el tamaño de gota y para un viento leve se fija la presión de trabajo a 3,5 bar con objeto de asegurar la presión nominal de funcionamiento del aspersor.

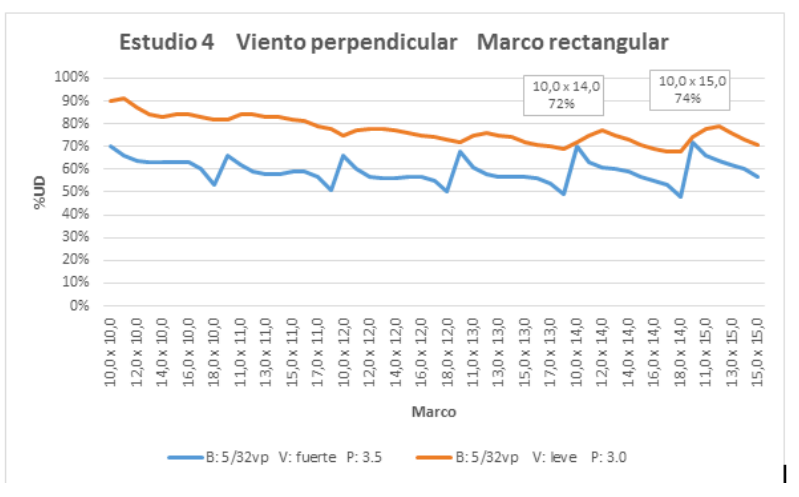

Gráfica 9: Gráfica lineal del porcentaje de uniformidad de distribución (UD) en diferentes tamaños de marco con dos tipos de velocidad de viento y presión de trabajo.

En condiciones de viento leve y presión de 3,5 bar se observa como la uniformidad de distribución decrece levemente conforme aumenta las dimensiones del marco y conforme crece la longitud de uno de sus lados.

En condiciones de viento fuerte y presión de 3,0 bar se observa como en ningún caso se obtienen valores de UD que lleguen al $75 \%$.

Se aprecia como en marcos tipo $10 \times 14$ o $10 \times 15$ se alcanza una UD cercana al $75 \%$, pero estos marcos son poco frecuentes y no atienden a una configuración estándar.

Para solucionar esta baja uniformidad de distribución habría q regar con poco viento o probar con una boquilla diferente.

\section{4- Conclusiones}

De este proyecto obtenemos en claro, lo útil y eficaz que es el banco de aspersión automatizado, el cual se convierte en una herramienta muy eficiente para solucionar el problema de la uniformidad en los sistemas de riego por aspersión.

Debido al ingente volumen de resultados que se obtienen de los ensayos realizados sería necesario diseñar una herramienta informática de consulta o un sistema experto, que con estos datos buscase la mejor solución a problemas particulares. De esta forma se podrían mejorar los recursos energéticos, utilizando los resultados obtenidos en el banco de ensayos para un funcionamiento aceptable del aspersor con presiones bajas de trabajo.

De los estudios presentados, se pueden obtener algunas conclusiones interesantes respecto a la eficiencia de aplicación del agua y uniformidad de riego bajo condiciones de viento cuando se emplea riego por aspersión. Estas conclusiones se afirman con los ensayos realizados hasta el momento, no se aseguran que con una casuística mayor se mantengan. Por tanto, y dada la eficiencia del sistema, interesaría seguir ensayando para la obtención de una volumen mayor de datos. Como resumen de todas las situaciones contempladas en el presente estudio, se ha observado que: 
La uniformidad de distribución mejora usando aspersores con dos boquillas respecto al uso de una sola boquilla.

La UD desciende más acusadamente al aumentar la velocidad del viento en marcos amplios respecto a separaciones menores

En aspersores con dos boquillas, los marcos cuadrados presentan mayor UD que los marcos rectangulares

Los marcos triangulares tienen mayor UD respecto a los marcos rectangulares

Dichas conclusiones coinciden y complementan lo expuesto por Tarjuelo Martín, Benito (1992)

\section{5-Bibliografía}

CAMPBELL SCIENTIFIC (1995): CR1000 Measurement and Control Modile. Instruction manual. Campbell Scientific. Inc. Leicester. UK.

DE JUAN, J. TARJUELO, J. VALIENTE, M. GARCIA, P. (1994): Non-uniformity and management of sprinkler irrigation systems. Proceedings of the third congress of the European Society for Agronomy, Padova University, Abano-Padova, Italy, 18-22 September 1994.; 1994. 102-103. 3 ref.

ESTUDILLO CASTRO, FRANCISCO (2013): Diseño de un banco de ensayos experimental automatizado para el análisis de los efectos de la dirección de ataque del viento.

FERNÁNDEZ GÓMEZ, RAFAEL (2010): Manual de riego para agricultores: modulo 3. Riego por aspersión.

ISO 7749-1:1986, Equipos de riego-Aspersores rotativos-Parte 1: Diseño y exigencias de funcionamiento.

ISO 7749-2:1990, Equipo de riego - Aspersores Rotativos - Parte 2: Uniformidad de distribución y métodos de prueba.

MERRIAN, J.L. y KELLER, J. (1978): Farm irrigation system evaluation: a guide for management. UTAH State University. Logan. Utah. USA

SALVATIERRA, B. (2010): Uniformidad de riego en las instalaciones de riego. Boletín trimestral SAR. No 14. Enero, marzo 2010.

SÁNCHEZ, I. FACI, J. M. ZAPATA, N. (2011): The effects of pressure, nozzle diameter and meteorological conditions on the performance of agricultural impact sprinklers. Agricultural Water Management; 2011. 102: 1, 13-24.

SEGINER, I. NIR, D. and BERNUTH, R. (1991): Simulation of wind-distorded sprinkler patterns. J. Irrig. Drain Eng., 117(2), 285-306

TARJUELO, J. M. CARRION, P. VALIENTE, M. (1994): Simulation of irrigation distribution by sprinkler under windy conditions. Investigacion Agraria, Produccion y Proteccion Vegetales; 1994. 9: 2, 255272. 15 ref.

TARJUELO MARTÍN BENITO, J. M. (1992): El riego por aspersión: diseño y funcionamiento. Albacete: Caja de Albacete; [Toledo]: Ediciones de la Universidad de Castilla-La Mancha, D.L.

TARJUELO MARTÍN BENITO, J. M. (2005): El riego por aspersión y su tecnología. Ediciones MundiPrensa. $581 \mathrm{pp}$.

VORIES, E. y VON BERNUTH, R. (1986): Single nozzle sprinkler performance in wind. Transactions of the ASAE, vol. 29 (5): 1325,1330.

ZAPATA, N. PLAYAN, E. MARTINEZ-COB, A. SANCHEZ, I. FACI, J. M. LECINA, S. (2008): From on-farm solid-set sprinkler irrigation design to collective irrigation network design in windy areas. Agricultural Water Management; 2007. 87: 2, 187-199. 37 ref. 